\title{
Origin of anomalously long interatomic distances in suspended gold chains
}

\author{
Sergio B. Legoas ${ }^{* 1}$, Douglas S. Galvão ${ }^{1}$, Varlei Rodrigues ${ }^{1,2}$, and Daniel Ugarte $^{2}$ \\ ${ }^{1}$ Instituto de Física "Gleb Wataghin", Universidade Estadual de Campinas, C.P. 6165, 13083-970 Campinas SP, Brazil and \\ ${ }^{2}$ Laboratório Nacional de Luz Síncrotron, C.P. 6192, 13084-971 Campinas SP, Brazil
}

(Dated: October 23, 2018)

\begin{abstract}
The discovery of long bonds in gold atom chains has represented a challenge for physical interpretation. In fact, interatomic distances frequently attain 3.0-3.6 $\AA$ values and, distances as large as 5.0 $\AA$ may be seldom observed. Here, we studied gold chains by transmission electron microscopy and performed theoretical calculations using cluster ab initio density functional formalism. We show that the insertion of two carbon atoms is required to account for the longest bonds, while distances above $3 \AA$ may be due to a mixture of clean and one $\mathrm{C}$ atom contaminated bonds.
\end{abstract}

PACS numbers: 68.65.-k, 61.46.+w, 68.37.Lp, 71.15.-m

In the last years considerable interest has been devoted to the study of linear gold atom chains (LAC)

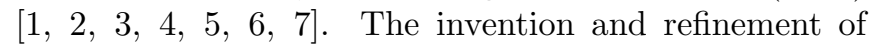
experimental techniques such as scanning tunneling microscope (STM) [1, 8, 9, 10], high resolution transmission electron microscopy (HRTEM) 田, 专, 11, 12, 13, 14, 15, and mechanically controllable break junction (MCBJ) [14, 16, 17] have made possible the fabrication of such atomic size contacts. As the LACs are in atomic scale, quantization of the conductance has been therefore observed (see recent review in [18]).

A surprising fact has been the observation of large bonds between gold atoms in stable monoatomic gold chains with respect to the normal nearest-neighbor distances in gold bulk $(2.88 \AA)$, or in $A_{2}$ dimers $(2.5 \AA)$ [19]. It has been reported [3, $4,5,14,20]$ that interatomic distances frequently vary in the 2.9 - $3.6 \AA$ range and also, extremely long distances close to $5 \AA$ have been rarely observed 15]. The origin of these large distances has been a serious and unresolved challenge for theoretical interpretation. Many theoretical studies have been reported using different techniques [6, 19, 21, 22] such as molecular dynamics and density functional theory (DFT). However, none of these works were able to reproduce the observed large interatomic distances. The chain rupture is always observed for distances significantly below the experimental values.

Neither of these theoretical works have considered the possibility of the monoatomic chains being contaminated by some impurities [4, 5, 14, 15] during sample fabrication. We have decided to explore this possibility, in special considering carbon atom contamination that, from experimental conditions, are most likely spurious atoms during HRTEM observations [5, 14. As recently demonstrated, the presence of carbon atoms would be undetectable (because of the low contrast) in HRTEM images

\footnotetext{
*Author to whom correspondence should be addressed. FAX:+5519-37885376. Electronic address:slegoas@ifi.unicamp.br
}

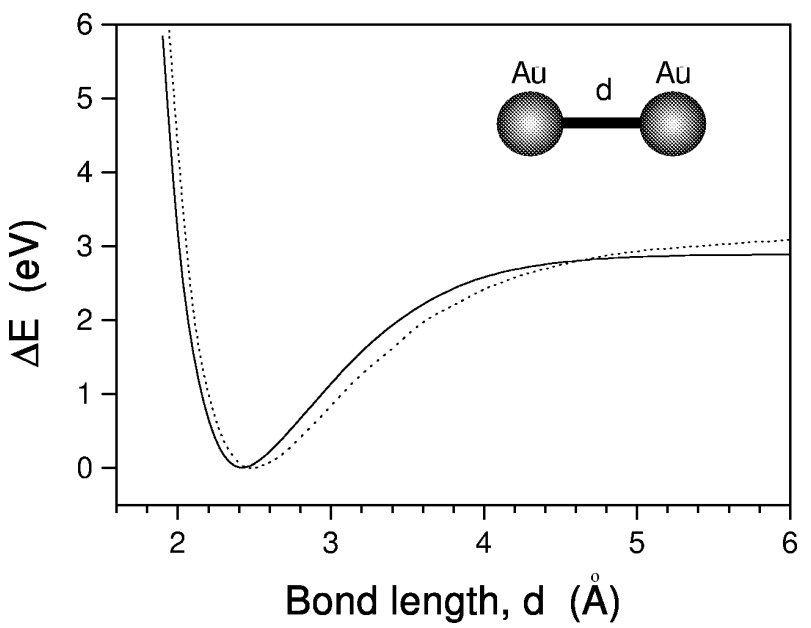

FIG. 1: $\mathrm{Au}_{2}$ total energy (relative to the minimum) as a function of the interatomic distance. Solid line (dot line) corresponds to calculation with DMol (Titan) package.

and would appear as large unusual $\mathrm{Au}-\mathrm{Au}$ distances [15].

In this paper we investigate both experimentally and theoretically the formation of suspended gold chains, addressing the calculations to analyze the origin of the large inter-gold distances experimentally observed.

We have generated stable linear gold atom chains in situ in a HRTEM (JEM 3010 URP, 300 kV, $1.7 \AA$ resol., at LME/LNLS Campinas, Brazil) following the method developed by Kondo and Takanayagi [13]. This procedure is based on using electron-beam irradiation (current density of $100 \mathrm{~A} / \mathrm{cm}^{2}$ ) to drill holes at different sites of a self-supported gold thin film ( $5 \mathrm{~nm}$ thick, average grain size $50-100 \mathrm{~nm}$ ) until a nanometric neck is formed between neighboring holes; the electron beam intensity is then reduced $\left(\sim 30 \mathrm{~A} / \mathrm{cm}^{2}\right)$ in order to perform the image acquisition. This experimental approach has been carefully described elsewhere [23]. The presented images were acquired from real time recording using a high sensitivity TV camera (Gatan 622SC) coupled to a conventional video recorder.

In order to try to understand the origin of the un- 


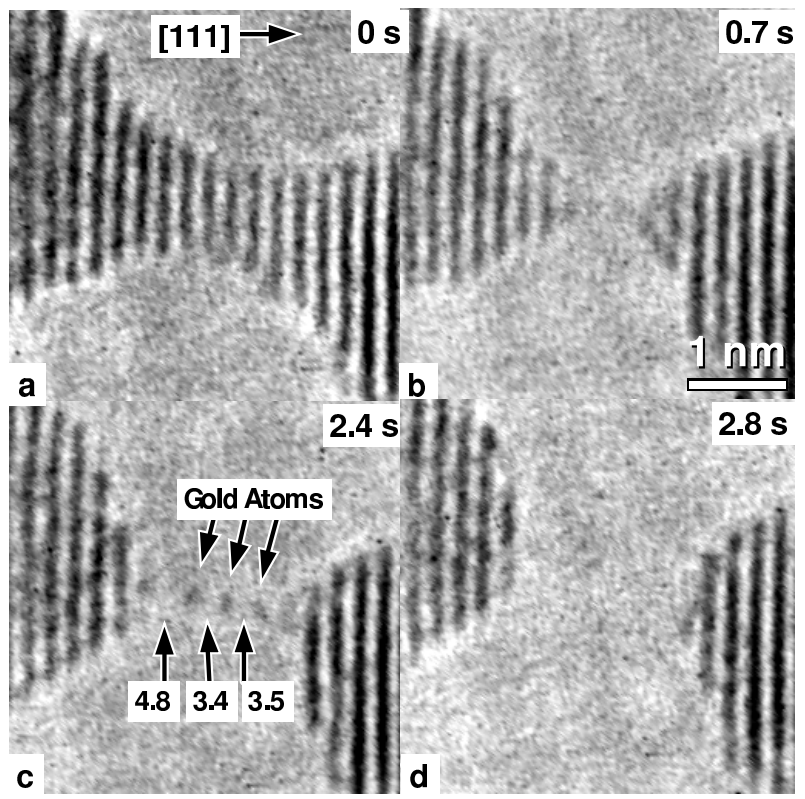

FIG. 2: (a)-(d) Nanowire time evolution when stretched approximately along the [111] direction. An LAC is formed in (c), where the $\mathrm{Au}-\mathrm{Au}$ interatomic distances are indicated in $\AA$ (see text for explanations). Atom positions appear dark.

usual large $\mathrm{Au}-\mathrm{Au}$ interatomic distances molecular clusters were used to simulate LACs. The geometry optimization calculations were performed using ab initio density functional total energy methods. We have used DMOL [24] and TITAN [25] packages. The density functional is treated by local density approximation with a Wang-Perdew [26] (Vosko-Wilk-Nusair) 27] exchangecorrelation functional for DMOL (TITAN) case. For the computation, we have used a double numerical (DND) basis set together with polarization functions for DMOL, and a LACVP** basis set in the case of TITAN. The reliability and the method dependence of the predicted geometries were tested carrying out a comparative study for the dimeric interdistance. As we can see from Fig. 1 , the qualitative features are almost the same and the minimum predicted distance $(2.44 \AA$ for DMOL, and 2.49 $\AA$ for TITAN calculations) are in very good agreement with the experimental value of $2.47 \AA$ [28, 29].

In Fig. 2 we show a series of snapshots of the elongation of a gold nanowire. After forming a one-atomthick constriction in Fig. 2b, further stretching induces the generation of an LAC consisting of three hanging atoms (Fig. 2(c)); finally, the constriction breaks and the apexes retract (Fig. 2(d)). This atom chain has been chosen because it displays a high contrast and also a peculiar interatomic distance distribution: a) two bonds within the frequently reported range (3.4 and $3.5 \AA$, error bar $0.2 \AA$ ) and; b) a extremely long bond attaining $4.8 \AA$. Bonds in the 4.5-5 $\AA$ interval have already been reported [13], but although they do occur, they have been

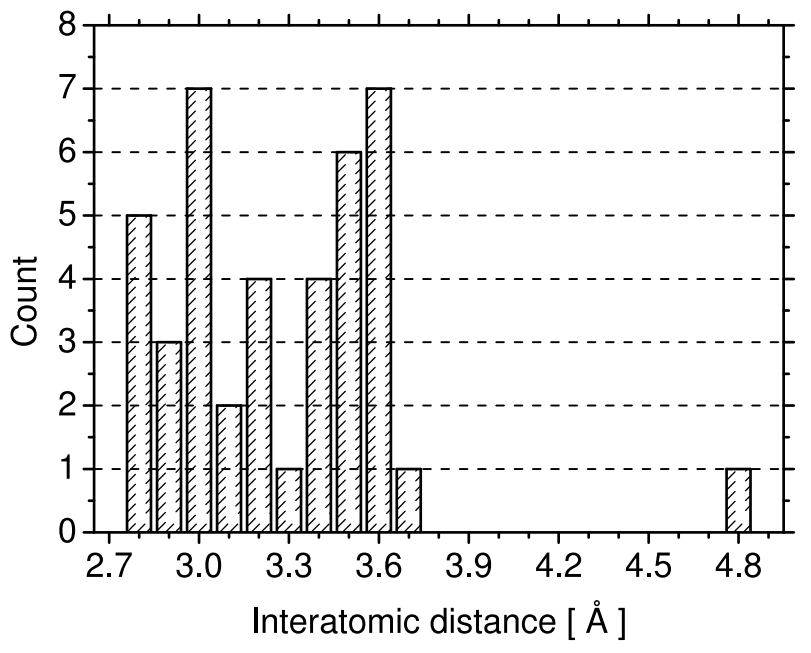

FIG. 3: Histogram on bond-lengths measured between hanging atom in gold LACs. A total of 41 different distances are included. The analyzed LACs were generated from wires elongated along different crystallographic directions [14].

observed only in very rare cases in our HRTEM experiments. It is interesting to observe the LAC interatomic distance histogram distribution (Fig. 3). Although the number of counts is rather low, we can easily observe that the bond-length values cover the whole range from 2.88 $\AA$ (nearest neighbor distance) to $3.7 \AA$. The histogram shows a peak position at about $3.6 \AA$, which seems to be a threshold value. In addition, an isolated point is measured at $4.8 \AA$, corresponding to the image in Fig. $2(\mathrm{c})$.

The image in Fig. 2c, allows the clear identification of two quite different interatomic distances which coexist on the same LAC. Thus, they can not be related to the same phenomena, as for example tensile stress on clean gold atoms. This example suggests that the presence of contamination is one of the main issues to be analyzed, in particular considering that light atoms such as carbon would not be detectable within the signal-to-noise ratio of typical HRTEM images 15. We have then proceeded to model the LAC structures considering the presence of carbon contamination. Since it has also been speculated that other atoms could be present [15], we have analyzed $\mathrm{N}, \mathrm{O}, \mathrm{S}$, and $\mathrm{Si}$ atoms as well. However, carbon is the atom that provides results more consistent when compared to the experimental data. Thus we present here only the $\mathrm{C}$ results.

Initial structures were generated considering that the gold atoms are at arbitrary positions forming linear chains at distances of $\sim 4.0 \AA$. The carbon atoms were positioned at distances of $\sim 1.5 \AA$ above the middle chain positions. Geometrical optimizations were then carried out. No constraints or symmetries were imposed. We have adopted the standard "naked" cluster approach, where no hydrogen or dummy termination atoms are used 
[30, 31]. Recent ab-initio DFT calculations for suspended gold chains [6] showed that the geometries for "naked" finite and infinite (cyclic boundary conditions) structures are essentially the same. This is an indication that the "naked" approach is appropriated for LACs.

In Fig. 4, we present the results for some selected structures obtained with DMOL; TITAN calculations produced similar results. The displayed examples are representative of possible structures considering the inclusion of carbon atoms at different positions or configurations. We have considered $\mathrm{C}$ incorporation both in its atomic and dimeric forms. In the former case, $\mathrm{C}-\mathrm{Au}$ and the $\mathrm{Au}-\mathrm{Au}$ distances are of 1.8 and $3.6 \AA$, respectively, and the structures are almost linear [Fig. 4(b)]. It is interesting to notice that the presence of a $\mathrm{C}$ atom between gold atoms does not affect their next $\mathrm{Au}-\mathrm{Au}$ distances [Fig. 4(c)] which remains at the same value of the $\mathrm{Au}_{2}$ [Fig. 4(a)]. The same behavior is observed for the $\mathrm{C}_{2}$ incorporation, with the exception that the $\mathrm{Au}-\mathrm{Au}$ distances are now $\sim 5.0 \AA$ [Figs. 4(d) and 4(e)]. To verify whether the presence of a large number of terminal gold atoms would affect these bond-lengths, we have also considered the case of adding two pyramidal (4 atoms each) gold structures [Fig. 4(f)] at the chain termination. As we can see, the distance pattern is almost the same of Fig. 4(e).

In Figs. 4(g) and 4(h), we show the obtained theoretical structures in two situations, totally free to relax and with the length geometrical constraint to match the total LAC length as observed in the experimental chain reported in Fig. 3 of Ref. [5]. For Fig. 4(g) the values are almost the same for similar structures considered in Figs. 4(b) and 4(c). For the constrained structure [Fig. 4(h)], we observe that the distances are slightly affected $(0.1 \AA$ reduction $)$ and can be attributed to the observed bending. Considering the experimental error bar $(0.2 \AA)$, the agreement between the experimental and theoretical data is excellent, providing strong support to the presence of $\mathrm{C}$ atoms as proposed. In Fig. 4(i) we show a predicted structure for the case of an unstrained linear chain of four gold atoms including dimeric and atomic $\mathrm{C}$ incorporation. This system is to be compared with the LAC of Fig. 2(c). Again, considering the error bar there is an excellent match between the experimental and theoretical data.

The theoretical calculations presented above have mainly addressed the effect of carbon incorporation into the suspended atom chains. We would like to stress that the existence of distances in the range of 3.0-3.6 $\AA$ do not necessarily demand the existence of $\mathrm{C}$ impurities. This can be deduced from the bond-length histogram in Fig. 3 which shows (within available low statistics) a continuous range from the expected gold distance in the bulk form to $3.7 \AA$, as it should be expected for tensile stress elongation applied on clean bonds. However the histogram peak at about $3.5 \AA$ could result as a direct effect of the C pres- (a)

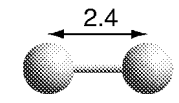

(b)

(c)

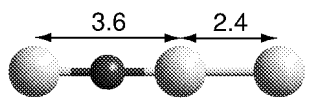

(d)

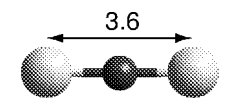

(e)

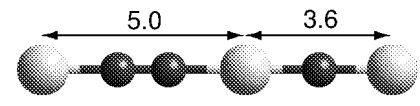

(f)

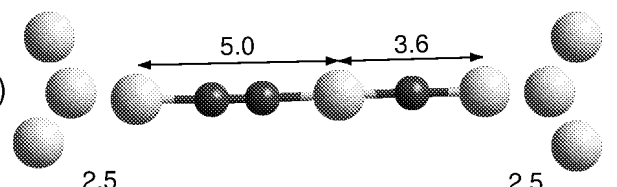

(g)

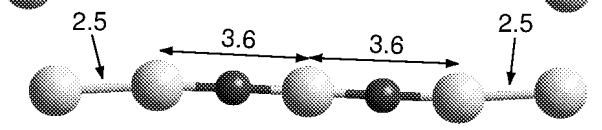

(h)

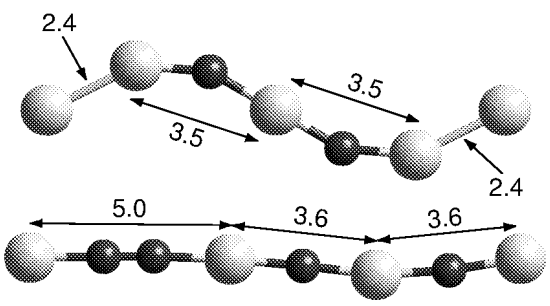

FIG. 4: Optimized gold chains with carbon impurities, for selected configurations. The chain (a) is a gold dimer. The other chains, except (f), are bidimensional molecules, contained in the figure plane. The system in (f) is a gold-carbon chain, with suspended tips in the extremes, composed by four gold atoms in a near (100)-Au geometry. Light (dark grey) spheres correspond to gold (carbon) atoms in the chain. Distances are in $\AA$.

ence. An alternative explanation for the more frequent observation of $3.5 \AA$ interatomic distance can be that this value is the threshold for elongation [32] and, further increase would require larger tensile stress. At this point, the apexes start to absorb the elastic energy because they have larger degrees of freedom, as predicted in molecular dynamic simulations 33, 34. Thus, the more frequent occurrence of the $3.6 \mathrm{~A}$ bonds is due to the fact that this length has a longer life time and then allow better imaging conditions during the experiments. The experimental data do not allows us to rule out the two situations (clean or contaminated). However, the histogram profile seems to be more compatible with the coexistence of the two regimes.

As for the longest $\mathrm{Au}-\mathrm{Au}$ distances of $\sim 4.0-5.0 \AA$ our results strongly indicate that they should not exist (as evidenced by many theoretical works) [6, 19, 21, 22]. To explain these distances we must invoke the presence of impurity contamination, more specifically $\mathrm{C}_{2}$. There are many possible sources of contamination in transmission electron microscopy work, such as vacuum oil from rotatory and diffusion pumps, grease and rubber O-rings, etc.. Concerning the physical origin, radiation damage 
of adsorbed hydrocarbon molecules causes a carbon rich, polymerized film to form and grow on the electron irradiated areas (for details, see for instance Ref. [35]). In our experiments the initial carbon layer is cleaned by the intense irradiation (see Refs. 15, 14, 23, however during the nanowire rupture the vacuum chamber contains hydrocarbon molecules from the residual gas pressure (10-7 torr in our HRTEM). Although these molecules are in low density, they slowly and continuously deposit carbon atoms on the nanowires surface. When these $\mathrm{C}$ atoms are generated close to the junction region (where the e-beam is focused) they may be incorporated into the suspended atom chains. The remarkable agreement between experimental and our theoretical data, associated with the fact that the presence of $\mathrm{C}$ atoms during the experiment is highly probable and should not be detectable, support the structures we are proposing.

In summary, we have experimentally observed the formation of stable linear gold atom chains, and get direct real-space information on atomic positions and bondlengths. To explain the origin of large gold-gold interdistances in those linear chains, we have carried out $a b$ initio density functional theory geometrical optimization calculations for gold clusters assuming the presence of $\mathrm{C}$ atoms as impurity contamination. Our results show that the apparent puzzle of the existence of long interatomic Au-Au distances (4.0-5.0 $\AA$ ) can be easily explained by the presence of $\mathrm{C}_{2}$ impurities. However, the bond-lengths above $3.0 \AA$ might be due to a mixture of clean stressed bonds and those contaminated by incorporating a single carbon atom. In principle these same arguments are applicable to other metallic monoatomic nanowires (formed under similar experimental conditions) such as Pt 36. and Ag [37, whose structures should also present large intermetallic distances.

Work supported in part by the Brazilian agencies CNPq, FINEP, and FAPESP. The authors also wish to acknowledge support from Accelrys, Inc. for helpful assistance.

[1] U. Landman et al., Science 248, 454 (1990).

[2] U. Landman, W.D. Luedtke, B.E. Salisbury, and R.L. Whetten, Phys. Rev. Lett. 77, 1362 (1996).

[3] A.I. Yanson et al., Nature 395, 783 (1998).

[4] H. Ohnishi, Y. Kondo, and K. Takayanagi, Nature 395, 780 (1998).

[5] V. Rodrigues and D. Ugarte, Phys. Rev. B 63, 073405 (2001).

[6] D. Sánchez-Portal et al., Phys. Rev. Lett. 83, 3884 (1999).
[7] T. Kizuka, S. Umehaa and S. Fujisawa, Jpn. J. Appl. Phys. 240, L71 (2001).

[8] L. Olesen et al., Phys. Rev. Lett. 72, 2251 (1994).

[9] N. Agraït, J.G. Rodrigo, and S. Vieira, Phys. Rev. B 47, 12345 (1993).

[10] J.J. Pascual et al., Phys. Rev. Lett. 71, 1852 (1993).

[11] T. Kizuka et al., Phys. Rev. B 55, R7398 (1997).

[12] T. Kizuka, Phys. Rev. Lett. 81, 4448 (1998).

[13] Y. Kondo and K. Takayanagi, Bull. Am. Phys. Soc. 44, 312 (1999).

[14] V. Rodrigues, T. Fuhrer, and D. Ugarte, Phys. Rev. Lett. 85, 4124 (2000).

[15] H. Koizumi, Y. Oshima, Y. Kondo, and K. Takayanagi, Ultramicroscopy 88, 17 (2001).

[16] C.J. Muller, J.M. van Ruitenbeek, and L.J. de Jongh, Physica C 191, 485 (1992).

[17] J.M. Krans et al., Nature (London) 375, 767 (1995).

[18] J.M. van Ruitenbeek in Metal Clusters at Surfaces, edited by K.-H. Meiwes-Broer, Cluster Series (Springer-Verlag Berlin Heidelberg, New York, 2000).

[19] H. Häkkinen, R.N. Barnett, and U. Landman, J. Phys. Chem. B 103, 8814 (1999).

[20] Y. Takai et al., Phys. Rev. Lett. 87, 106105 (2001).

[21] M.R. Sørensen, M. Brandbyge, and K.W. Jacobsen, Phys. Rev. B 57, 3283 (1998).

[22] M. Okamoto and K. Takayanagi, Phys. Rev. B 60, 7808 (1999).

[23] V. Rodrigues and D. Ugarte, Europ. J. Phys. D 16, 395 (2001).

[24] B. Delley, J. Chem. Phys. 92, 508 (1990); B. Delley, J. Chem. Phys. 113, 7756 (2000). DMol3 is available from Accelrys Inc. as part of Materials Studio and the Cerius2 program suites. http://www.accelrys.com.

[25] Titan is a program suite from Wavefunction, Inc. http://www.wavefun.com.

[26] Y. Wang and J.P. Perdew, Phys. Rev. B 43, 8911 (1991).

[27] S.J. Vosko, L. Wilk, and M. Nusair, Can. J. Phys. 58, 1200 (1980).

[28] O. D. Häberlen, S.C. Chung, and N. Rösch, Int. J. Quantum Chem. Symp. 28, 595 (1994).

[29] B.D. Yu and M. Scheffler, Phys. Rev. B 56, R15 569 (1997).

[30] O. D. Häberlen, S.C. Chung, M. Stener, and N. Rösch, J. Chem. Phys. 106, 5189 (1997).

[31] G. Pacchioni, S.C. Chung, S. Krüger, and N. Rösch, Chem. Phys. 184, 125 (1994).

[32] P. Pyykkö and Y. Zhao, Angew. Chem. Int. Ed. Engl. 30, 604 (1991).

[33] E.Z. da Silva, A.J.R. da Silva, and A. Fazzio, Phys. Rev. Lett. 87, 256102 (2001).

[34] G. Rubio-Bollinger et al., Phys. Rev. Lett. 87, 026101 (2001)

[35] Ludwig Reimer, Transmission Electron Microscopy, Springer Series in Optical Sciences, ed. Peter W. Hawkes, 4th edition, Springer Verlag, Berlin, 1997.

[36] V. Rodrigues and D. Ugarte, submitted.

[37] V. Rodrigues et al., submitted. 